\title{
A multicenter, open-label, expanded phase 2 study to evaluate the safety and efficacy of etirinotecan pegol, a polymer conjugate of irinotecan, in women with recurrent

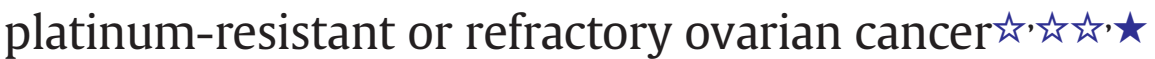

\author{
G. Rustin ${ }^{\text {a }}$, I. Vergote ${ }^{\mathrm{b}}$, J.P. Micha ${ }^{\mathrm{c}}$, L.R. Duska ${ }^{\mathrm{d}}$, N. Reed ${ }^{\mathrm{e}}$, J. Bendell ${ }^{\mathrm{f}}$, D. Spitz ${ }^{\mathrm{g}}$, G. Dark ${ }^{\mathrm{h}}$, U. Hoch ${ }^{\mathrm{i}}$, \\ M. Tagliaferri ${ }^{i}$, A.L. Hannah ${ }^{\mathrm{i}}$, A.A. Garcia ${ }^{\mathrm{j}, \mathrm{k}, *}$ \\ a Mount Vernon Hospital, Middlesex, United Kingdom \\ ${ }^{\mathrm{b}}$ Universitair Ziekenhuis Leuven, Leuven, Belgium \\ c Gynecologic Oncology Associates, Newport Beach, CA, USA \\ d University of Virginia Health System, Division of Gynecologic Oncology, Charlottesville, VA, USA \\ e Beatson West of Scotland Cancer Centre, Gartnavel General Hospital, Glasgow, United Kingdom \\ f Tennessee Oncology, PLLC, Sarah Cannon Research Institute, Nashville, TN, USA \\ ${ }^{g}$ Palm Beach Cancer Institute, West Palm Beach, FL, USA \\ h Freemen Hospital, High Heaton, Newcastle-upon-Tyne, United Kingdom \\ i Nektar Therapeutics, San Francisco, CA, USA \\ ${ }^{j}$ USC Norris Comprehensive Cancer Center and Hospital, Los Angeles, CA, USA \\ ${ }^{\mathrm{k}}$ LSU Stanley S. Scott Cancer Center, New Orleans, LA, USA
}

\section{H I G H L I G H T S}

- Heavily pretreated platinum-resistant ovarian cancer (PROC) population.

- Encouraging activity with etirinotecan pegol (EP): ORR 15\%, median PFS 4.4 mo, median OS 10.2 mo.

- Adverse events related to EP were manageable with supportive care.

- Activity of EP in this population (especially in patients with PROC who received prior PLD) warrants continued development.

\section{A R T I C L E I N F O}

\section{Article history:}

Received 22 May 2017

Received in revised form 15 August 2017

Accepted 24 August 2017

Available online $\mathrm{xxxx}$

\section{Keywords:}

Ovarian cancer

Etirinotecan pegol

Platinum-resistant

Platinum-refractory

\section{A B S T R A C T}

Objective. Etirinotecan pegol (EP) is a novel polyethylene glycol conjugated form of irinotecan with documented activity in platinum-resistant ovarian cancer (PROC). We report the results of the expanded portion of a phase II study of EP in patients with PROC who received prior pegylated liposomal doxorubicin (PLD) or who were unable to receive it.

Methods. This multicenter, open-label, phase II study evaluated EP q21d for PROC. The primary endpoint was objective response rate (ORR) by Response Evaluation Criteria in Solid Tumors version 1.0. Secondary endpoints included progression-free survival (PFS), overall survival (OS), and safety. Patient populations evaluated included a modified intent-to-treat (mITT) group consisting of all patients who received at least one dose and with measurable disease and a primary efficacy ( $\mathrm{pEFF}$ ) group (subset of the mITT population who received prior PLD).

Results. One hundred thirty-nine patients were enrolled. Of the 132 patients in the mITT group, 20 achieved an ORR (15.2\%; 95\% CI 9.5-22.4); median PFS and OS were 4.4 months and 10.2 months, respectively. In the pEFF group ( $\mathrm{n}=104), 15$ patients $(14.4 \%$; 95\% CI 8.3-22.7) achieved an ORR; median PFS and OS were 4.4 months and 10.9 months, respectively. The most common grade $3 / 4$ toxicities were diarrhea (20\%), abdominal pain (17\%), vomiting (14\%), dehydration (13\%), and nausea (13\%). Severe diarrhea was reduced to $15 \%$ with strict adherence to screening and management guidelines.

Conclusions. This study confirms the activity and safety of single-agent EP in patients with PROC, including patients who received prior PLD. Further evaluation earlier in the disease course and in combination is warranted.

(c) 2017 Published by Elsevier Inc.

is This study was registered at ClinicalTrials.gov, number NCT00806156.

论动 Research support: Nektar Therapeutics.

$\star$ Funding: Nektar Therapeutics.

* Corresponding author at: Louisiana State University, 533 Bolivar Street, New Orleans, LA 70112, USA.

E-mail address: agarc6@lsuhsc.edu (A.A. Garcia). 


\section{Introduction}

The choice of chemotherapy for recurrent ovarian cancer is usually based on the interval between the completion of last platinum-based treatment and the detection of relapse, the platinum-free interval (PFI) [1]. Typically, patients with platinum refractory or resistant ovarian cancer (PROC) have low response rates to subsequent non platinumbased chemotherapy (10-20\%) lasting only 3-4 months, with subsequent median survival of approximately one year [2-5]. There is a need for better treatments for this group of patients.

Etirinotecan pegol (EP), a long-acting topoisomerase I inhibitor, is a novel polyethylene glycol conjugated form of irinotecan engineered to reduce peak irinotecan and SN38 (the major active metabolite of irinotecan) plasma concentrations, which may be responsible for unwanted cholinergic reactions and severe myelosuppression, yet provide prolonged systemic and increased tumor exposure to SN38 [6-10]. The significant unmet need in the treatment of PROC in addition to the promising early preclinical and clinical trials prompted investigation of EP in a phase II study aimed at better defining its clinical activity in ovarian cancer. As previously published, EP was evaluated in two dosing schedules ( $145 \mathrm{mg} / \mathrm{m}^{2}$ every 14 days [q14d] or every 21 days [q21d] in a randomized phase II trial in patients with PROC [11]. Both EP schedules provided encouraging activity in this heavily-pretreated population (with the median number of prior regimens of 3 ), with a confirmed overall response rate (ORR) in approximately $20 \%$ of patients. Median progression-free survival (PFS) and overall survival (OS) were numerically higher, although not statistically significant, for the q21d regimen compared with the q14d regimen (5.3 and 11.7 months and 4.1 and 10.0 months, respectively). An exploratory assessment of a subset of patients that received prior pegylated liposomal doxorubicin (PLD) suggested EP activity in this population, with confirmed ORR of $21 \%$ in the q21d regimen and median PFS of 5.4 months [12]. The most commonly reported grade 3 to 4 adverse events (AEs) were dehydration (24\%) and diarrhea $(23 \%)$, with a $31 \%$ rate of grade $3 / 4$ diarrhea in the q14d regimen and $14 \%$ in the q21d regimen. Similarly, fewer patients in the q21d arm experienced other grade 3/4 toxicities including dehydration, nausea, hypokalemia, and hyponatremia. Grade 3-4 hematologic toxicity reported in the q14d and q21d schedules included neutropenia (14\% and $9 \%$ ) and febrile neutropenia ( $11 \%$ and $0 \%$ ), respectively.

Based on better tolerability, a trend toward improved PFS and OS, and the convenience of less frequent dosing, the every-21-day treatment schedule of EP $145 \mathrm{mg} / \mathrm{m}^{2}$ was selected for expanded study to further elucidate its clinical activity and improved tolerability. In addition, we aimed to further define the activity and safety of EP in patients with PROC by separately evaluating those patients who had previously received PLD.

\section{Patients and methods}

\subsection{Study design and patients}

This was a multicenter, open-label, phase II study of EP in patients with metastatic or unresectable locally advanced PROC. The study consisted of two portions: a randomized portion (in PROC patients), as previously reported [11], and an expansion portion (in PROC patients who received prior PLD therapy in a platinum-resistant setting or for whom treatment with PLD was contraindicated). Patients were enrolled from December 2008 until February 2012 at 23 sites in three countries ( 5 sites in Belgium, five sites in the UK, and 13 sites in the USA).

Inclusion criteria included the following: women aged $\geq 18$ with histologically or cytologically confirmed inoperable metastatic or locally advanced epithelial ovarian cancer, primary peritoneal cancer, or fallopian tube cancer; measurable disease by Response Evaluation Criteria in Solid Tumors (RECIST; version 1.0) [13] in $\geq 1$ lesion not previously irradiated; platinum-resistant disease (defined as progression by RECIST within 6 months of last dose of most recent platinum drug); Eastern Cooperative Oncology Group (ECOG) performance status of 0 or 1 ; and adequate renal, hepatic, and hematologic function. Eligibility for the expansion group was restricted to patients with PROC with disease progression following PLD therapy or who were otherwise unable to receive further PLD therapy. There was no upper limit to the number of prior lines of therapy a patient could have received.

Patients were excluded from the study if they had received chemotherapy or radiotherapy within 4 weeks of study drug initiation and had not recovered to an associated National Cancer Institute Common Terminology Criteria for Adverse Events (NCI-CTCAE) version 3.0 toxicity of grade 1 (with any grade alopecia allowed and no grade of diarrhea allowed); had major surgery within 4 weeks or minor surgery within 2 weeks of study drug initiation; received prior treatment with a camptothecin derivative (e.g., topotecan, irinotecan, or any SN38 investigational drug); had a history of hypersensitivity to other pegylated drugs; received an investigational drug or biologic agent (e.g., bevacizumab) within 28 days of study drug initiation; received selected cytochrome P450 3A4 inhibitors or inducers within 2 weeks prior to study drug initiation or during the study; had known or suspected central nervous system metastases; were pregnant or lactating; or had other significant comorbid conditions that in the opinion of the investigator would impair study participation or cooperation.

In order to improve safety and compliance with the recommended diarrhea screening and management guidelines outlined within the study, an amendment was implemented in March 2011 to mandate full resolution of diarrhea (defined as grade 0 by NCI-CTCAE for at least 7 days without supportive antidiarrheal measures, including antidiarrheal medications) prior to redosing EP. Additionally, sites were mandated to proactively screen for diarrhea and institute aggressive management for any occurrence.

The study was conducted in accordance with local institutional review boards and/or independent ethics committees and all patients provided signed informed consent prior to study entry.

\subsection{Procedures}

Patients reported herein received EP administered at a dose of $145 \mathrm{mg} / \mathrm{m}^{2}$ as a 90 -minute intravenous infusion on day 1 of each treatment cycle every 21 days (data for patients who received EP every 14 days was previously reported) [17]. Treatment was continued until disease progression, unacceptable toxicity, death, withdrawal by patient, principal investigator decision, or other criteria (e.g., lost to follow-up, protocol violation, study termination by sponsor). Dosage modifications were recommended based on hematologic and nonhematologic parameters. A new treatment cycle was not initiated until recovery to an absolute neutrophil count $\geq 1500 / \mu \mathrm{L}$, platelet count $\geq 100,000 / \mu \mathrm{L}$, hemoglobin value $\geq 9.0 \mathrm{~g} / \mathrm{dL}$, grade $\leq 2$ nonhematologic toxicity, and full resolution of treatment-related diarrhea for at least $48 \mathrm{~h}$ without supportive antidiarrheal measures (this was increased to 7 days approximately half-way through the study to improve the safety of redosing EP with its extended half-life). Prophylactic antidiarrheal medication was not allowed for either treatment group; however, patients were instructed to have loperamide readily available and to begin treatment at the first episode of poorly formed or loose stools or the earliest onset of bowel movements more frequent than normally expected for the patient. Treatment modifications for subsequent cycles were based on nadir (hematologic) or peak (nonhematologic) values accordingly; no dose re-escalation was allowed after a dose reduction.

Tumor measurements were documented using computed tomography (CT) scans or magnetic resonance imaging (MRI) and were performed at screening, approximately every 6 weeks during the treatment period, and at the end-of-treatment visit (if not assessed within the previous 28 days). Scans after the end-of-treatment visit may have been collected if the patient had not progressed or had not received new anticancer treatment. The same method of assessment (CT or MRI) and the same technique for acquisition of data was used to 
characterize each lesion at each measurement. Investigator assessment of scans was the primary method for ORR classification. A centralized imaging service was utilized as confirmation for sensitivity analyses. Routine laboratory testing and assessment of AEs were performed at screening, at the beginning of every treatment cycle, and at the endof-treatment visit. The end-of-treatment visit occurred within $30 \pm$ 3 days after last dose of study drug. Approximately every 3 months following the end-of-treatment visit, patients were contacted to assess disease progression, survival, subsequent anticancer therapy, and resolution of all toxicity attributable to study drug. Quarterly follow-up continued until death, withdrawal by patient or at the discretion of the principal investigator and/or physician, lost to follow-up, or study terminated by the sponsor.

\subsection{Outcomes}

The following patient populations were used to evaluate the study objectives: 1) modified intent-to-treat (mITT) population: all patients meeting criteria for PROC who received at least one dose (or partial dose) of study drug (q21d regimen) and had measurable disease by RECIST; 2) primary efficacy (pEFF) population: a subgroup of the mITT group who received prior PLD therapy in a platinum-resistant setting or who were otherwise unable to receive further PLD therapy; 3 ) platinum-refractory population: patients with platinum-refractory ovarian cancer defined as a PFI of $\leq 6$ weeks after any line of platinum. The safety analysis was based on the mITT population.

The primary objective was ORR by RECIST guidelines for patients in the mITT, pEFF, and platinum-refractory populations. Secondary objectives included duration of response (DoR), best ORR by Gynecologic Cancer InterGroup (GCIG) guidelines ( $\geq 50 \%$ reduction in CA-125 levels from a pretreatment sample confirmed and maintained for at least 28 days; pretreatment level must be twice the upper limit of normal within 2 weeks of starting treatment) [14],clinical benefit rate (complete response, partial response, or stable disease [ $\geq 3$ months] by RECIST), PFS, OS, 6-month and/or 1-year survival rates, and incidence and duration of toxicities according to NCI-CTCAE version 3.0. In addition, efficacy and safety endpoints were assessed in patients pre- and post-amendment on diarrhea, which mandated full resolution for at least 7 days prior to redosing of EP.

\subsection{Statistical analysis}

The sample size for the second stage of the randomization portion of the study was based upon the null hypothesis of $p \leq 0.050$ and the alternative hypothesis of $p \geq 0.200$, where $p$ is proportion of response in each treatment arm; the type 1 error was 0.029 (target 0.050), and type 2 error was 0.145 (target 0.20 ). The objective of the expansion of the study was to provide accurate estimate of ORR for patients with PROC who had received prior PLD in a platinum-resistant setting or who were otherwise unable to receive further PLD; approximately 145 patients were deemed to be necessary for expansion to obtain at least 100 patients for the primary efficacy analysis. Two-sided $95 \%$ confidence intervals $(\mathrm{CI})$ for ORR based on the Clopper-Pearson methodology were calculated. Data were tabulated and analyzed with respect to patient enrollment and disposition, demographic and baseline characteristics, prior and concomitant medications, as well as efficacy and safety measures. Exploratory analyses to examine efficacy endpoints by prognostic factors were also performed. Time-to-event endpoints were summarized using Kaplan-Meier methodology, medians, CIs, and quartiles.

\section{Results}

From December 2008 to February 2012,139 patients received EP q21d in the phase II trial. Among them, 35 patients were included in the randomization portion; of these, 6 patients started treatment with
$170 \mathrm{mg} / \mathrm{m}^{2}$ prior to dose reduction by protocol amendment due to current safety data. Disposition of study participants is outlined in Fig. 1 and Table 1. Demographic and baseline disease characteristics are outlined in Table 2. The median age of patients was 59 years; $52 \%$ of patients had an ECOG performance status of 1, and $64 \%$ had serous histology. All patients except 4 had measurable disease per RECIST at baseline per investigator's assessment. The median times from the initial diagnosis to the first dose of study drug and from the primary diagnosis of metastatic disease were 2.4 years and 2.1 years, respectively. Overall, patients were heavily pretreated, with almost two-thirds of patients (68\%) receiving 3 or more prior cytotoxic regimens and 72\% receiving two or more prior platinum-based regimens; median number of prior cytotoxic regimens was three (range, 1-18) and prior platinum-based therapies was two (range, 1-10). Almost all patients (98\%) received taxane-containing therapy, and $84 \%$ of patients received prior PLD therapy. The median PFI was approximately 11 weeks; $39 \%$ of patients met the criteria for platinum-refractory disease ( $\mathrm{PFI} \leq 6$ weeks).

Overall, 20 patients (15.2\%; 95\% CI, 9.5-22.4) had a confirmed ORR per investigator assessment in the mITT population $(n=132)$; median PFS and OS were 4.4 months (95\% CI, 2.9-5.0) and 10.2 months (95\% CI, 8.2-12.2), respectively (Table 3 ). These patients had a median DoR of 6.6 months and a CBR of $50.8 \%$; ORR by GCIG criteria was $25 \%$. Results were similar in the pEFF $(n=104)$ and platinum-refractory populations $(n=52)$; GCIG response was lower for the refractory population. Estimated 12 -month OS was $43.1 \%$ in the mITT population and $36.9 \%$ in platinum-refractory population.

All 139 patients were included in the safety population. Patients received a median of 4 treatment cycles (Table 1 ). The most common treatment-emergent AEs $(\geq 20 \%)$ included diarrhea (76\%), nausea (78\%), fatigue (58\%), and vomiting (55\%) (Table 4). Grade 3 diarrhea occurred in 20\% of patients; no grade 4 diarrhea was reported (Table 4 ).

Twenty-three patients (17\%) discontinued study drug due to an $\mathrm{AE}$; one of these patients started treatment at $170 \mathrm{mg} / \mathrm{m}^{2}$ (Supplemental Table 1 ). Overall, $70 \%$ of patients required a dose delay and $82 \%$ a dose reduction due to an $\mathrm{AE}$. The most common $\mathrm{AE}$ leading to study drug discontinuation was diarrhea (6\%). One death was thought to be at least partially attributable to study drug. This patient had peritoneal metastases and prior to her death was noted to have an intestinal obstruction due to a large tumor infiltrating her bowel. She had received 5 doses of EP $145 \mathrm{mg} / \mathrm{m}^{2}$. Starting 13 days after her last dose, the patient experienced fever with increasing abdominal distention followed by neutropenia and impaired kidney function. Despite aggressive treatment measures, the patient died 101 days after initiating treatment with EP.

An amendment requiring full resolution of diarrhea for at least 7 days before reinitiation of EP was implemented during the study. Supplemental Table 2 depicts patient characteristics, efficacy parameters, and toxicity associated with the regimen prior to and after implementation of amendment 5. Baseline patient and disease characteristics were similar between amendment 4 and 5 populations except for shorter PFI in amendment 5 patients ( 14 weeks and 10 weeks, respectively). All patients were previously treated with $\operatorname{PLD}(n=97)$. After amendment implementation, there was an increase in treatment exposure duration (91 vs 108.5 days in amendment 4 and 5 populations, respectively) and median number of cycles received ( 4 vs 5 cycles, respectively). The frequency of grade 3 diarrhea was reduced from $26 \%$ with amendment 4 to $15 \%$ with amendment 5 . The median number of days of grade 3 diarrhea was reduced from 10 days to 4.8 days, and the percent of patients permanently stopping the drug due to diarrhea was $9 \%$ vs $5 \%$ in the amendment 4 and 5 populations, respectively. The amendment 5 population had similar efficacy parameters, including an ORR of $18 \%$, median PFS of 4.6 months, and OS of 11.5 months.

Exploratory subgroup analyses for all efficacy endpoints were conducted in the mITT population including platinum resistance [refractory ( $\mathrm{PFI} \leq 6$ weeks) and relatively refractory ( $\mathrm{PFI}>6$ weeks to 6 months)], the number of prior platinum-based chemotherapies, and the number of prior cancer therapy regimens (Table 5). As depicted, ORR was 


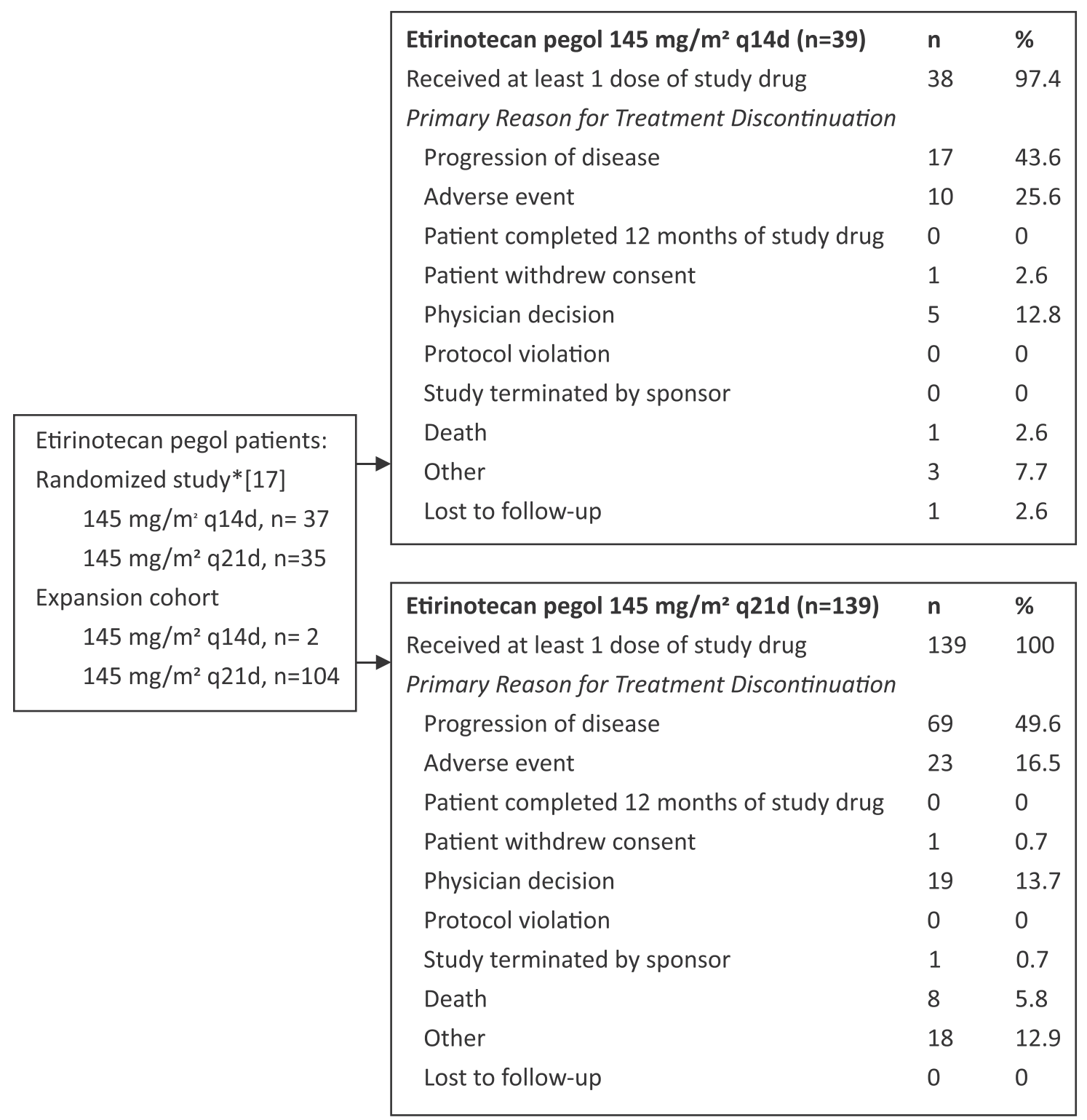

Fig. 1. CONSORT diagram. *Randomized study previously published.

maintained across subsets and did not trend lower in patients with more refractory disease or in patients who received multiple lines of prior therapy. Shorter median PFS and OS were estimated in patients with PFI $\leq 6$ weeks; however, PFS and OS were increased in patients who received a greater number of prior platinum or other therapies. Further analyses of patients who received $\geq 3$ prior cytotoxic regimens ( $n=90)$ as well as those who received $\geq 3$ prior cytotoxic regimens and $\geq 2$ prior platinum regimens $(n=81)$ demonstrated that these subgroups achieved ORRs, PFS, and OS consistent with what was observed in the MITT and pEFF populations.

Other analyses included comparison of characteristics of responding patients $(n=24)$ by investigator review to patients who did not achieve a response $(n=145)$. The two groups were generally comparable with minor exceptions: median baseline CA-125 and incidence of patients with ECOG 1 were higher in the responding group; last PFI was longer in the responding group; 2 patients in the nonresponding group received prior camptothecin therapy compared to no patients in the responding group. Given the small sample size for responding patients, no definitive conclusions can be made.

\section{Discussion}

The primary purpose of this expanded phase II trial was to further define the activity of EP administered every 21 days in patients with PROC. The findings reported herein, in which EP was administered as a fourth-line regimen for most patients, demonstrated an ORR of $15 \%$, clinical benefit achieved in over half of the patients, and a median PFS and OS of 4.4 months and 10.2 months, respectively. Compared to other single cytotoxic agents used in advanced PROC, including PLD, topotecan, and gemcitabine, these results are encouraging [2,4,5,15-19]. Additionally, these response rates and median PFS and OS were similar in women with PROC who had failed or could not receive treatment with PLD. In this setting, there is no accepted standard of care. The addition of bevacizumab to topotecan, PLD, or paclitaxel has expanded available treatment options for women with recurrent PROC, particularly showing a pronounced treatment effect in combination with weekly paclitaxel; however, many PROC patients cannot receive paclitaxel because of prior paclitaxel induced neuropathy [20,21]. 
Table 1

Patient disposition.

\begin{tabular}{ll}
\hline & Etirinotecan $145 \mathrm{mg} / \mathrm{m}^{2} \mathrm{q} 21 \mathrm{~d}$ \\
\hline Enrolled & 139 \\
Randomized portion & 35 \\
Expansion portion & 104 \\
Started at dose level & \\
$170 \mathrm{mg} / \mathrm{m}^{2}$ & 6 \\
$145 \mathrm{mg} / \mathrm{m}^{2}$ & 133 \\
Median number of cycles received (range) & $4.0(1,20)$ \\
Safety population & 139 \\
mITT population $^{\mathrm{a}}$ & 132 \\
pEFF population & \\
Platinum-refractory population &
\end{tabular}

Abbreviations: mITT, modified intent-to-treat; pEFF, primary efficacy population; $\mathrm{PFI}$, platinum-free interval; PLD, pegylated liposomal doxorubicin; PROC, platinum-resistant ovarian cancer.

a All patients with PROC that met eligibility criteria; excluded 7 patients (did not have measureable disease or did not meet study entry criteria for platinum-resistant disease).

b Patients treated q21d and had received prior PLD in platinum-resistant setting or were unable to receive further PLD; excluded patients from mITT population who did not receive PLD or it was not given in platinum-resistant setting.

c Patients in mITT group $(n=132)$ with a PFI $\leq 6$ weeks.

It is important to critically examine the studied populations when indirectly comparing the results of this trial to those of other agents in the published literature. Platinum-resistant disease is this study was defined as progression by RECIST within 6 months of last dose of most recent platinum drug (not following a patient's primary treatment with platinum); platinum-refractory was similarly defined as PFI (time to recurrence/progression from last dose of the prior platinum-based therapy) of $\leq 6$ weeks. These definitions concurred with recommendations from the Gynecologic Cancer InterGroup consensus on clinical trials in recurrent ovarian cancer that distinct patient populations for clinical trial enrollment be based on the interval from last platinum therapy and on the time to progression [22].

This was a heavily-pretreated population where two-thirds of the patients enrolled had received $\geq 3$ prior regimens and almost $40 \%$ were deemed as having platinum-refractory disease, which has an extraordinarily poor prognosis and such patients are often excluded from clinical trials. Additionally, 70\% of patients had received 2 or more prior platinum-based regimens. An encouraging and notable finding from this trial was the consistency of the results reported across all efficacy parameters in all populations studied, including the platinum-refractory population in which $15 \%$ of patients achieved a confirmed ORR by RECIST (19\% by GCIG criteria), disease control was observed in 43\%, and median PFS and OS were 3 months and 9.4 months, respectively. When the data in the mITT population were further analyzed based on well-established prognostic factors including sensitivity to platinum, ORR was similar between platinum-refractory patients (15\%) and relatively-resistant patients (17\%), (defined as PFI $>6$ weeks but $\leq 6$ months). The consistency of response was also observed among different subsets of patients based upon the number of prior cancer therapies and the number of prior platinum-based therapies.

Despite the fact that patients in this expanded phase II trial had previously received multiple prior chemotherapy regimens, treatment with EP was generally well tolerated and adverse events manageable with prompt initiation of supportive care. The most common grade 3 / 4 AEs observed were diarrhea, dehydration, and fatigue. Grade 3 or higher neutropenia, anemia, and thrombocytopenia were infrequently reported. The study was amended mandating that diarrhea must resolve to a grade 0 prior to redosing EP. As observed, the institution of this amendment allowed patients to receive more study drug, observed by both the increase in exposure duration and median number of cycles received; and importantly, provided a reduction in the incidence of grade 3 or greater toxicities, with a reduction by almost one-half of grade 3 diarrhea, from $26 \%$ to $15 \%$ pre- and post-amendment,
Table 2

Baseline patient and tumor characteristics.

\begin{tabular}{|c|c|}
\hline \multirow[t]{2}{*}{ Characteristic } & \multirow{2}{*}{$\begin{array}{l}\text { All enrolled patients } \\
\text { Etirinotecan } 145 \mathrm{mg} / \mathrm{m}^{2} \mathrm{q} 21 \mathrm{~d} \\
(n=139)\end{array}$} \\
\hline & \\
\hline \multicolumn{2}{|l|}{ Age, years } \\
\hline Median & 59 \\
\hline Range & 21,80 \\
\hline$\geq 65$, no. (\%) & $40(29)$ \\
\hline \multicolumn{2}{|l|}{ ECOG PS, no. (\%) } \\
\hline 0 & $66(48)$ \\
\hline 1 & $72(52)$ \\
\hline 2 & $1(1)$ \\
\hline Measurable disease by RECIST, no. (\%) & $135(97)$ \\
\hline \multicolumn{2}{|l|}{ Time since initial diagnosis, years } \\
\hline Median & 2.4 \\
\hline Range & $0.5,20.8$ \\
\hline \multicolumn{2}{|l|}{ Time since diagnosis of metastatic disease, years } \\
\hline Median & 2.1 \\
\hline Range & $0,16.1$ \\
\hline \multicolumn{2}{|l|}{ Histologic subtype, no. (\%) } \\
\hline Malignant clear cell & $9(7)$ \\
\hline Malignant Brenner & $1(1)$ \\
\hline Malignant mixed epithelial tumor & $4(3)$ \\
\hline Mixed & $3(2)$ \\
\hline Other & $30(22)$ \\
\hline Serous & $89(64)$ \\
\hline Mucinous & $3(2)$ \\
\hline \multicolumn{2}{|l|}{ Prior cancer regimens, no. (\%) } \\
\hline Median & 3 \\
\hline Range & 1,18 \\
\hline 1 & $6(4)$ \\
\hline 2 & $39(28)$ \\
\hline 3 & $41(30)$ \\
\hline $4+$ & $53(38)$ \\
\hline \multicolumn{2}{|l|}{ Prior platinum-based regimens, no. (\%) } \\
\hline Median & 2 \\
\hline Range & 1,10 \\
\hline 1 & $39(28)$ \\
\hline 2 & $54(39)$ \\
\hline 3 & $31(22)$ \\
\hline $4+$ & $15(11)$ \\
\hline \multicolumn{2}{|l|}{ Time to progression from last platinum, weeks ${ }^{\mathrm{a}}$} \\
\hline Median & 11 \\
\hline Range & $-5,67$ \\
\hline Platinum-free interval, $\leq 6$ weeks, no. (\%) & $54(39)$ \\
\hline $\begin{array}{l}\text { Platinum-free interval, }>6 \text { weeks }-\leq 6 \text { months, } \\
\text { no. (\%) }\end{array}$ & $85(61)$ \\
\hline \multicolumn{2}{|l|}{ Prior therapy, no. (\%) } \\
\hline PLD & $117(84)$ \\
\hline Taxane & $136(98)$ \\
\hline Gemcitabine & $51(37)$ \\
\hline Bevacizumab & $18(13)$ \\
\hline
\end{tabular}

Note: Six patients received $170 \mathrm{mg} / \mathrm{m}^{2}$ which was lowered to $145 \mathrm{mg} / \mathrm{m}^{2}$.

Abbreviations: ECOG PS, Eastern Cooperative Oncology Group performance status; PFI, platinum-free interval; PLD, pegylated liposomal doxorubicin; RECIST, Response Evaluation Criteria In Solid Tumors.

a Determined from date of last dose of platinum to disease progression.

respectively. The median number of days of grade 3 diarrhea was reduced (from 10 to 4.8 days), as was the percent of patients permanently stopping the drug due to diarrhea (from 5 to 2 patients). Importantly, delaying retreatment until diarrhea had settled did not impact the efficacy of EP; and in fact, efficacy parameters in the post-amendment population were slightly improved, most likely attributable to the improved tolerability.

As the vast majority of patients do not respond to contemporary agents once their disease has become resistant to platinum agents, there is a critical need for the development of innovative and effective therapeutic strategies. EP represents a novel therapy for ovarian cancer as noted by confirmed activity in platinum-resistant and -refractory disease, particularly in patients who had previously received PLD treatment and regardless of the number of prior therapies received, 
Table 3

Primary and secondary efficacy outcomes in studied populations.

\begin{tabular}{|c|c|c|c|}
\hline & $\begin{array}{l}\text { mITT population } \\
(n=132)\end{array}$ & $\begin{array}{l}\text { pEFF population } \\
(n=104)\end{array}$ & $\begin{array}{l}\text { Platinum-refractory } \\
\text { population }^{\mathrm{a}}(n=52)\end{array}$ \\
\hline \multicolumn{4}{|l|}{ RECIST } \\
\hline ORR & $20(15.2 \%)$ & $15(14.4 \%)$ & $8(15.4 \%)$ \\
\hline$[95 \% \mathrm{CI}]$ & {$[9.5,22.4]$} & {$[8.3,22.7]$} & {$[6.9,28.1]$} \\
\hline DoR (median) & $6.6 \mathrm{mo}$ & $7.4 \mathrm{mo}$ & $7.4 \mathrm{mo}$ \\
\hline $\mathrm{CBR}^{\mathrm{b}}$ & $67(50.8 \%)$ & $53(51.0 \%)$ & $20(38.5 \%)$ \\
\hline \multicolumn{4}{|l|}{$\mathrm{GCIG}^{\mathrm{c}}$} \\
\hline ORR & $33(25.0 \%)$ & $26(25.0 \%)$ & $9(17.3 \%)$ \\
\hline$[95 \% \mathrm{CI}]$ & {$[17.9,33.3]$} & {$[17.0,34.4]$} & {$[8.2,30.3]$} \\
\hline \multicolumn{4}{|l|}{ PFS } \\
\hline $\begin{array}{l}\text { Progressed or died } \\
\text { on study, } \mathrm{n}\end{array}$ & $102(77.3 \%)$ & $80(76.9 \%)$ & $40(76.9 \%)$ \\
\hline Median & $4.4 \mathrm{mo}$ & $4.4 \mathrm{mo}$ & $2.7 \mathrm{mo}$ \\
\hline$[95 \% \mathrm{CI}]$ & {$[2.9,5.0]$} & {$[2.9,5.0]$} & {$[1.7,4.6]$} \\
\hline \multicolumn{4}{|l|}{ OS } \\
\hline Died on study, n & $90(68.2 \%)$ & 69 (66.3\%) & $36(69.2 \%)$ \\
\hline Median & $10.2 \mathrm{mo}$ & $10.9 \mathrm{mo}$ & $8.0 \mathrm{mo}$ \\
\hline$[95 \% \mathrm{CI}]$ & {$[8.2,12.2]$} & {$[8.2,13.1]$} & {$[5.0,12.2]$} \\
\hline 12-month & $43.1 \%$ & $44.3 \%$ & $36.9 \%$ \\
\hline$[95 \% \mathrm{CI}]$ & {$[34.1 \%, 51.9 \%]$} & {$[34.0 \%, 54.1 \%]$} & {$[23.3 \%, 50.5 \%]$} \\
\hline
\end{tabular}

Abbreviations: $\mathrm{CBR}$, clinical benefit rate; $\mathrm{CI}$, confidence interval; $\mathrm{CR}$, complete response; DoR, duration of response; GCIG, Gynecologic Cancer InterGroup; mITT, modified intentto-treat; ORR, overall response rate; OS, overall survival; pEFF, primary efficacy population; PFS, progression-free survival; PR, partial response; RECIST, Response Evaluation Criteria in Solid Tumors; SD, stable disease; ULN, upper limit of normal.

a Defined as a platinum-free interval of $\leq 6$ weeks.

b Defined as CR + PR + SD [ $\geq 3$ months].

c Response defined as a $\geq 50 \%$ reduction in CA-125 levels from a pretreatment sample (level at least $2 x U L N$ ); must be confirmed and maintained for at least 28 days.

whether platinum-based or not. As such, the demonstrable activity noted from EP in this heavily-pretreated PROC population warrants continued development of the drug, both as a single agent as well as in combination with other active agents (eg, bevacizumab, carboplatin, PLD, weekly paclitaxel) and novel targeted therapies.

\section{Role of the funding source}

This study was supported by funding from Nektar Therapeutics. The funding source was involved in the study design and collection, analysis, and interpretation of the data. The principal investigator and leading coinvestigators had input in the design of the study, did the study,

Table 4

Most common ( $\geq 20 \%$ ) treatment-emergent adverse events and grades $\geq 3$.

\begin{tabular}{|c|c|c|c|c|}
\hline \multirow[t]{2}{*}{ Adverse event } & \multicolumn{4}{|c|}{$\begin{array}{l}\text { Safety population }{ }^{\mathrm{a}} \\
(n=139)\end{array}$} \\
\hline & All grades & Grade 3 & Grade 4 & Grade 5 \\
\hline Diarrhea & $105(76 \%)$ & $28(20 \%)$ & - & - \\
\hline Dehydration & $32(23 \%)$ & $18(13 \%)$ & $1(1 \%)$ & - \\
\hline Fatigue & $81(58 \%)$ & $22(16 \%)$ & $1(1 \%)$ & - \\
\hline Abdominal pain & $78(56 \%)$ & $23(17 \%)$ & - & - \\
\hline Nausea & $108(78 \%)$ & $18(13 \%)$ & - & - \\
\hline Vomiting & 77 (55\%) & $20(14 \%)$ & - & - \\
\hline Decreased appetite & $69(50 \%)$ & $17(12 \%)$ & - & - \\
\hline Neutropenia & $27(19 \%)$ & $7(5 \%)$ & $2(1 \%)$ & - \\
\hline Hypokalemia & $28(20 \%)$ & $7(5 \%)$ & - & - \\
\hline Anemia & $39(28 \%)$ & $9(7 \%)$ & $1(1 \%)$ & - \\
\hline Small intestinal obstruction & $12(9 \%)$ & $8(6 \%)$ & $2(1 \%)$ & $1(1 \%)$ \\
\hline Constipation & $51(37 \%)$ & $3(2 \%)$ & $1(1 \%)$ & - \\
\hline Weight decreased & $42(30 \%)$ & - & - & - \\
\hline Alopecia & $29(21 \%)$ & - & - & - \\
\hline
\end{tabular}

a Defined as all enrolled patients who received at least one dose (or partial dose) of the study drug.
Table 5

Subgroup analyses.

\begin{tabular}{|c|c|}
\hline & mITT population $(n=132)$ \\
\hline \multicolumn{2}{|l|}{ RECIST confirmed ORR/evaluable } \\
\hline $\mathrm{PFI}^{\mathrm{a}} \leq 6$ weeks (refractory) & $8 / 52(15 \%)$ \\
\hline$>6$ weeks $-\leq 6$ mo., (resistant) & $11 / 79(14 \%)$ \\
\hline \multicolumn{2}{|l|}{ Prior lines of platinum } \\
\hline 1 & $5 / 37(14 \%)$ \\
\hline 2 & $8 / 51(16 \%)$ \\
\hline$\geq 3$ & $7 / 44(16 \%)$ \\
\hline \multicolumn{2}{|l|}{ Prior no. previous regimens } \\
\hline 1 & $1 / 5(20 \%)$ \\
\hline 2 & $5 / 37(14 \%)$ \\
\hline 3 & $5 / 39(13 \%)$ \\
\hline 4 & $5 / 20(25 \%)$ \\
\hline$\geq 5$ & $4 / 31(13 \%)$ \\
\hline \multicolumn{2}{|l|}{ PFS, months } \\
\hline $\mathrm{PFI}^{\mathrm{a}} \leq 6$ weeks (refractory) & $2.7 \mathrm{mo}$ \\
\hline$>6$ weeks $-\leq 6$ months (resistant) & $4.7 \mathrm{mo}$ \\
\hline \multicolumn{2}{|l|}{ Prior lines of platinum } \\
\hline 1 & $2.9 \mathrm{mo}$ \\
\hline 2 & $4.4 \mathrm{mo}$ \\
\hline$\geq 3$ & $4.6 \mathrm{mo}$ \\
\hline \multicolumn{2}{|l|}{ Prior no. previous regimens } \\
\hline 1 & $1.9 \mathrm{mo}$ \\
\hline 2 & $2.9 \mathrm{mo}$ \\
\hline 3 & $3.9 \mathrm{mo}$ \\
\hline 4 & $6.8 \mathrm{mo}$ \\
\hline$\geq 5$ & $4.6 \mathrm{mo}$ \\
\hline \multicolumn{2}{|l|}{ OS, months } \\
\hline $\mathrm{PFI}^{\mathrm{a}} \leq 6$ weeks (refractory) & $8.0 \mathrm{mo}$ \\
\hline$>6$ weeks $-\leq 6$ months (resistant) & $11.0 \mathrm{mo}$ \\
\hline \multicolumn{2}{|l|}{ Prior lines of platinum } \\
\hline 1 & $8.9 \mathrm{mo}$ \\
\hline 2 & $11.6 \mathrm{mo}$ \\
\hline$\geq 3$ & $11.0 \mathrm{mo}$ \\
\hline \multicolumn{2}{|l|}{ Prior no. previous regimens } \\
\hline 1 & $7.4 \mathrm{mo}$ \\
\hline 2 & $9.5 \mathrm{mo}$ \\
\hline 3 & $9.4 \mathrm{mo}$ \\
\hline 4 & $17.7 \mathrm{mo}$ \\
\hline \multirow[t]{2}{*}{$\geq 5$} & $14.0 \mathrm{mo}$ \\
\hline & $\mathrm{q} 21 \mathrm{~d}(n=90)$ \\
\hline \multicolumn{2}{|l|}{ Received $\geq 3$ prior regimens } \\
\hline RECIST confirmed ORR & $14(16 \%)$ \\
\hline DoR (months) & 6.6 \\
\hline $\mathrm{CBR}^{\mathrm{b}}$ & $51(57 \%)$ \\
\hline GCIG confirmed ORR & $24(27 \%)$ \\
\hline PFS, median (months) & 4.6 \\
\hline OS, median (months) & 11.5 \\
\hline
\end{tabular}

Abbreviations: $C B R$, clinical benefit rate; $C R$, complete response; DoR, duration of response; GCIG, Gynecologic Cancer InterGroup; MITT, modified intent-to-treat; ORR, overall response rate; OS, overall survival; PFI, platinum-free interval; PFS, progression-free survival; PR, partial response; RECIST, Response Evaluation Criteria in Solid Tumors; SD, stable disease.

a Determined from date of last dose of platinum to disease progression.

b Defined as CR + PR + SD [ $\geq 3$ months].

provided oversight for the analysis and interpretation of the data, and worked in consultation with Nektar Therapeutics in the writing of this manuscript. The corresponding author and coauthors had access to all the trial data and had final responsibility for the decision to submit for publication.

\section{Acknowledgements}

We thank the patients and the investigators who participated in this study. We also thank Dr. Charles C. Pippitt (Piedmont Hematology Oncology Associates, Winston-Salem, NC, USA) and Prof. Christopher Poole (University Hospital Coventry \& Warwickshire, NHS Trust, Coventry, United Kingdom) for enrolling patients into the trial. We thank Phillips Gilmore Oncology Communications for providing medical writing support, funded by Nektar Therapeutics. 


\section{Appendix A. Supplementary data}

Supplementary data to this article can be found online at http://dx. doi.org/10.1016/j.ygyno.2017.08.026.

\section{References}

[1] D. Luvero, A. Milani, J. Ledermann, Treatment options in recurrent ovarian cancer: latest evidence and clinical potential, Ther. Adv. Med. Oncol. 6 (2014) 229-239.

[2] R. Coleman, B.J. Monk, A.K. Sood, T.J. Herzog, Latest research and treatment of advanced-stage epithelial ovarian cancer, Nat. Rev. Clin. Oncol. 10 (2013) 211-224.

[3] J.A. Ledermann, R.S. Kristeleit, Optimal treatment for relapsing ovarian cancer, Ann Oncol 21 (Suppl. 7) (2010) vii218-222.

[4] K.H. Baumann, U. Wagner, A. du Bois, The changing landscape of therapeutic strategies for recurrent ovarian cancer, Future Oncol. 8 (2012) 1135-1147.

[5] R.W. Naumann, R.L. Coleman, Management strategies for recurrent platinum-resistant ovarian cancer, Drugs 71 (2011) 1397-1412.

[6] C. Alemany, Etirinotecan pegol: development of a novel conjugated topoisomerase I inhibitor, Curr. Oncol. Rep. 16 (2014) 367-372.

[7] U. Hoch, C.M. Staschen, R.K. Johnson, M.A. Eldon, Nonclinical pharmacokinetics and activity of etirinotecan pegol (NKTR-102), a long-acting topoisomerase 1 inhibitor, in multiple cancer models, Cancer Chemother. Pharmacol. 74 (2014) 1125-1137.

[8] G.S. Jameson, J.T. Hamm, G.J. Weiss, et al., Multicenter, phase I, dose-escalation study to assess the safety, tolerability, and pharmacokinetics of etirinotecan pegol in patients with refractory solid tumors, Clin. Cancer Res. 19 (2013) 268-278.

[9] J.G. Slatter, L.J. Schaaf, J.P. Sams, et al., Pharmacokinetics, metabolism, and excretion of irinotecan (CPT-11) following I.V. infusion of [14C]CPT-11 in cancer patients, Drug Metab. Dispos. 28 (2000) 423-433.

[10] Y.C. Wang, C.C. Chang, K.J. Chiang, et al., Topoisomerase I inhibitor suppress tumor growth in chemoresistant ovarian cancer-initiating cells, J. Med. Sci. 34 (2014) 72-76.

[11] I.B. Vergote, A. Garcia, J. Micha, et al., Randomized multicenter phase II trial comparing two schedules of etirinotecan pegol (NKTR-102) in women with recurrent platinum-resistant/refractory epithelial ovarian cancer, J. Clin. Oncol. 31 (2013) 4060-4066.
[12] A.A. Garcia, I. Vergote, J.P. Micha, C.H. Pippitt, G.G. Rao, N. Spitz, et al., The role of NKTR-102 in women with platinum resistant/refractory ovarian cancer and failure on pegylated liposomal doxorubicin (PLD), Proc. Am. Soc. Clin. Oncol. 29 (suppl) (2011) (abstr 5047).

[13] P. Therasse, S.G. Arbuck, E.A. Eisenhauer, et al., New guidelines to evaluate the response to treatment in solid tumors: European Organisation for Research and Treatment of Cancer, National Cancer Institute of the United States, National Cancer Institute of Canada, J. Natl. Cancer Inst. 92 (2000) 205-216.

[14] G.J. Rustin, I. Vergote, E. Eisenhauer, et al., Definitions for response and progression in ovarian cancer clinical trials incorporating RECIST 1.1 and CA 125 agreed by the Gynecological Cancer Intergroup (GCIG), Int. J. Gynecol. Cancer 21 (2011) 419-423.

[15] N. Colombo, E. Kutarska, M. Dimopoulos, et al., Randomized, open-label, phase III study comparing patupilone (EPO906) with pegylated liposomal doxorubicin in platinum-refractory or -resistant patients with recurrent epithelial ovarian, primary fallopian tube, or primary peritoneal cancer, J. Clin. Oncol. 30 (2012) 3841-3847.

[16] M.L. Hensley, S. Kravetz, X. Jia, et al., Eribulin mesylate (halichondrin B analog E7389) in platinum-resistant and platinum-sensitive ovarian cancer: a 2-cohort, phase 2 study, Cancer 118 (2012) 2403-2410.

[17] M. Fung-Kee-Fung, T. Oliver, L. Elit, A. Oza, H.W. Hirte, P. Bryson, Optimal chemotherapy treatment for women with recurrent ovarian cancer, Curr. Oncol. 14 (2007) 195-208.

[18] K.J. O'Byrne, P. Bliss, J.D. Graham, et al., A phase III study of Doxil/Caelyx versus paclitaxel in platinum-treated, taxane naïve relapsed ovarian cancer, Proc. Am. Soc. Clin. Oncol. 21 (suppl) (2002) (abstr 808).

[19] A.N. Gordon, J.T. Fleagle, D. Guthrie, D.E. Parkin, M.E. Gore, A.J. Lacave, Recurrent epithelial ovarian carcinoma: a randomized phase III study of pegylated liposomal doxorubicin versus topotecan, J. Clin. Oncol. 19 (2001) 3312-3322.

[20] E. Pujade-Lauraine, F. Hilpert, B. Weber, et al., Bevacizumab combined with chemotherapy for platinum-resistant recurrent ovarian cancer: the AURELIA open-label randomized phase III trial, J. Clin. Oncol. 32 (2014) 1302-1308.

[21] A.M. Poveda, F. Selle, F. Hilpert, et al., Bevacizumab combined with weekly paclitaxel, pegylated liposomal doxorubicin, or topotecan in platinum-resistant recurrent ovarian cancer: analysis by chemotherapy cohort of the randomized phase III AURELIA trial, J. Clin. Oncol. 33 (2015) 3836-3838.

[22] M. Friedlander, E. Trimble, A. Tinker, et al., Clinical trials in recurrent ovarian cancer, Int. J. Gynecol. Cancer 21 (2011) 771-775. 